\title{
Construction de valeurs propres doubles du laplacien de Hodge-de Rham
}

\author{
Pierre Jammes
}

RÉSUMÉ.- Sur toute variété de dimension au moins 3, on construit une métrique telle que la première valeur propre non nulle du laplacien agissant sur les $p$-formes différentielles soit double. On en déduit qu'on peut prescrire le volume et le début du spectre du laplacien de Hodge-de Rham avec multiplicité 1 ou 2.

Mots-clefs : formes différentielles, laplacien de Hodge-de Rham, multiplicité de valeurs propres.

ABSTRACT.- - On any compact manifold of dimension greater than 3, we exhib a metric whose first positive eigenvalue for the Laplacian acting on $p$-form is of multiplicity 2. As a corollary, we prescribe the volume and any finite part of the spectrum of the Hodge Laplacian with multiplicity 1 or 2 .

Keywords : differential forms, Hodge Laplacian, multiplicity of eigenvalues.

MSC2000 : 58J50, 58C40

\section{Introduction}

Y. Colin de Verdière a montré dans [CdV86] que pour toute variété riemannienne compacte $M$ de dimension supérieure ou égale à 3 et tout entier $N \geq 1$, il existe une métrique sur $M$ telle que la multiplicité de la première valeur propre du laplacien agissant sur les fonctions de $M$ soit égale à $N$, et a généralisé ce résultat en montrant qu'on peut en fait prescrire toute partie finie du spectre du laplacien, la multiplicité des valeurs propres pouvant être choisie arbitrairement (voir [CdV87]).

Le problème de la multiplicité des valeurs propres a aussi été étudié pour des opérateurs de Schrödinger ([CdVT93], [BCC98]), et les résultats de prescription de spectre a été adaptés par P. Guérini au laplacien de Hodgede Rham - agissant sur les formes différentielles — dans Gu04 et par M. Dahl à l'opérateur de Dirac (Da05). Mais pour ces deux derniers opérateurs les valeurs propres prescrites sont simples (en se restreignant par exemple aux formes coexactes de degré fixé en ce qui concerne le laplacien de 
Hodge-de Rham); le problème de créer des valeurs propres multiples sur une variété quelconque dans ces deux cas n'a pas encore été résolue.

Le but de cet article est d'apporter un début de réponse à cette question en expliquant comment construire des métriques telles qu'une valeur propre du laplacien de Hodge-de Rham soit de multiplicité 2. La construction assure que cette multiplicité possède une certaine stabilité, ce qui permet finalement de prescrire le début du spectre avec multiplicité 1 ou 2 .

Comme dans Ja06b, on ne cherchera à prescrire le spectre du laplacien qu'en restriction aux formes coexactes. En effet, si on note

$$
0<\mu_{p, 1}(M, g) \leq \mu_{p, 2}(M, g) \leq \ldots
$$

les valeurs propres du laplacien agissant sur les $p$-formes coexactes de $M$, le spectre non nul du laplacien agissant sur l'ensemble des $p$-formes est $\left(\mu_{p-1, i}(M, g)\right)_{i \geq 1} \cup\left(\mu_{p, i}(M, g)_{i \geq 1}\right)$, et la multiplicité de la valeur propre nulle, si elle est existe, est le $p$-ième nombre de Betti de $M$. Comme la dualité de Hodge impose que $\mu_{p, i}(M, g)=\mu_{n-p-1, i}(M, g)$, on peut se restreindre aux degrés $p \leq[(n-1) / 2]$.

Théorème 1.2 Soit $M$ une variété compacte, connexe et orientable de dimension $n=2 k+1$ ou $2 k+2$ où $k \in \mathbb{N}^{*}, V$ un réel strictement positif et $N \geq 1$ un entier. On se donne pour tout entier $p \in\{1, \ldots, k\}$ une suite de réels $0<\nu_{p, 1} \leq \nu_{p, 2} \leq \ldots \leq \nu_{p, N}$, chaque valeur apparaissant au plus deux fois pour $p$ donné.

Il existe une métrique $g$ sur $M$ telle que

- $\mu_{p, i}(M, g)=\nu_{p, i}$ pour tout $i \leq N$ et $p \in\{1, \ldots, k\}$;

- $\operatorname{Vol}(M, g)=V$.

La construction de valeurs propres doubles s'appuiera sur l'apparition d'un phénomène spectral lié à la présence de multiplicité, baptisé « diabolo » dans [BW84, dont on peut par exemple trouver la description dans Ar76] (appendice 10) et [CdV98 (chapitre 5).

Dans CdV88, Y. Colin de Verdière formalise une notion de transversalité remontant à Arnol'd et définit deux propriétés de transversalité — resp. forte et faible, que nous rappellerons dans la section 4 - pour les valeurs propres multiples d'une forme quadratique. Il remarque dans CdV98 que dans le cas où l'hypothèse forte est vérifiée pour une multiplicité 2 , on peut montrer que cette multiplicité est stable mettant en évidence un point diabolo. D'une certaine manière, notre démarche sera inverse : on va construire un point diabolo sans faire intervenir d'hypothèse de transversalité, et on pourra vérifier a posteriori que la valeur propre double vérifie l'hypothèse faible de transversalité. 
Il serait bien sûr intéressant de savoir si on peut construire des valeurs propres de multiplicité plus grande sur toute variété. La technique que nous utiliserons est cependant spécifique à la multiplicité 2 et ne semble pas pouvoir s'adapter à des multiplicités plus grandes (voir remarque 4.5). Il convient toutefois de remarquer que sur certaines variétés, la multiplicité de la première valeur propre peut être arbitrairement grande :

Théorème 1.3 Pour tout entier $n \geq 4$, et tout $1 \leq p<n / 2$, il existe une variété $M$ de dimension $n$ telle que pour tout entier $k \geq 1$, il existe une métrique $g$ sur $M$ telle que $\mu_{p, 1}(M, g)$ soit de multiplicité au moins $k$.

En général, on ne peut donc pas majorer la multiplicité de la première propre en fonction de la topologie comme c'est le cas sur les surfaces. Mais les exemples du théorème 1.3 ont une topologie particulière (variétés produits) et on ne contrôle la multiplicité de la première valeur propre que pour certains degrés qu'on ne peut pas choisir indépendamment de la topologie. En ce sens, le théorème 1.2 qui prescrit les premières valeurs propres avec multiplicité 1 ou 2 pour tous les degrés simultanément et sur n'importe quelle variété est beaucoup plus précis.

Ces résultats permettent de mieux cerner le problème de la multiplicité des valeurs propres du laplacien de Hodge-de Rham et de dégager quelques questions qui restent en suspens, par exemple :

Question 1.4 La multiplicité de la première valeur propre de la sphère peutelle être arbitrairement grande quel que soit le degré?

Question 1.5 Si $M$ est une variété de dimension 3, la multiplicité de $\mu_{1,1}(M, g)$ est-elle nécessairement bornée? Si oui, comment varie la multiplicité maximale de $\mu_{1,1}(M, g)$ en fonction de la topologie?

La section 2 sera consacrée au rappel des outils techniques que nous utiliserons. Dans les sections 3 et 4 nous expliquerons comment construire une valeur propre double, et pourquoi sa multiplicité est stable. Enfin, dans les sections 5 et [ 6 , nous démontrerons les théorèmes 1.2 et 1.3 .

\section{Convergence de valeurs propres et d'espaces pro- pres}

Nous allons rappeler ici les outils techniques qui vont intervenir dans la construction de valeurs propres doubles. Le premier est le résultat de convergence de valeurs propres et d'espaces propres obtenu par C. Anné et B. Colbois dans AC95 pour les variétés compactes reliées par des anses fines : on se donne une famille finie de variétés compactes $\left(M_{j}, g_{j}\right)_{i=1}^{K}$ qu'on 
relie entre elles par des anses fines, isométriques au produit d'une sphère $\left(S^{n-1}, \varepsilon^{2} g_{\text {can }}\right)$ par un intervalle. En notant $\left(\tilde{M}, g_{\varepsilon}\right)$ la variété obtenue, qui est difféomorphe à $M_{1} \# M_{2} \# \ldots \# M_{K}$, on a alors :

Théorème 2.1 Si, pour $p \in\{1, \cdots, n-1\}$, on note $\mu_{p, 1}^{\prime} \leq \mu_{p, 2}^{\prime} \leq \ldots$ la réunion des spectres $\left(\mu_{p, i}\left(M_{j}, g_{j}\right)\right)_{i, j}$, on a pour tout $i \in \mathbb{N}^{*}$

$$
\lim _{\varepsilon \rightarrow 0} \mu_{p, i}\left(\tilde{M}, g_{\varepsilon}\right)=\mu_{p, i}^{\prime}
$$

et il y a convergence des espaces spectraux.

Ce théorème a déjà été utilisé pour prescrire le spectre du laplacien de Hodgede Rham sans multiplicité dans [Gu04] et Ja06b].

Pour obtenir de la multiplicité, on aura besoin d'un contrôle sur la vitesse de convergence des valeurs propres et des espaces propres. Ce contrôle découle de la proposition 3.10 ainsi et des corollaires 3.11 et 3.12 de AC95. (voir aussi les propositions 1 et 2 de [An90. Nous renvoyons aussi à An90 pour une définition précise de la convergence des espaces propres). Si $I$ est un intervalle de $\mathbb{R}^{+}, E_{I}^{\varepsilon}$ l'espace engendré par les $p$-formes propres de $\left(\tilde{M}, g_{\varepsilon}\right)$ de valeur propre contenue dans $I$ et $E_{I}$ l'espace engendré par les $p$-formes propres des $\left(M_{j}, g_{j}\right)$ de valeur propre contenue dans $I$, la distance entre $E_{I}$ et $E_{I}^{\varepsilon}$ vérifie $d\left(E_{I}, E_{I}^{\varepsilon}\right) \leq C \tau(\varepsilon)$ avec $\lim _{\varepsilon \rightarrow 0} \tau(\varepsilon)=0$ et où $C$ est une constante dépendant des bornes de $I$ et de leur distance au spectre $\left(\mu_{p, i}^{\prime}\right)_{i}$. En particulier, si deux valeurs propres de $\left(\tilde{M}, g_{\varepsilon}\right)$ sont proches l'une de l'autre mais assez éloignées du reste du spectre, il est difficile de localiser les formes propres mais la somme des deux espaces propres converge rapidement.

Ces résultats seront appliqués à la variété obtenue en attachant à $M$ des sphères munies de métriques bien choisies. Plus précisément, on utilisera les 《haltères de Cheeger généralisées » définies par P. Guérini dans [Gu04: pour tous entiers $n \geq 3$ et $1 \leq p \leq n-1$ et tout réel $u>0$ petit, on considère le domaine $\Omega_{p, u}$ de $\mathbb{R}^{n+1}$ formé par la réunion d'un $\frac{1}{5}$-voisinage tubulaire de la sphère unité $S^{p} \subset R^{p+1} \times\{0\} \subset \mathbb{R}^{n+1}$ et du produit de boules $B^{p+1}(0,1) \times B^{n-p}(0, u) \subset \mathbb{R}^{p+1} \times \mathbb{R}^{n-p}$. Après lissage de son bord, le domaine $\Omega_{p, u}$ est difféomorphe à une boule $B^{n+1}$ et $\partial \Omega_{p, u}$ est une sphère (voir la section 2.1 de Gu04 pour les détails de la construction). La famille de métriques induites sur le bord a la propriété de produire une petite valeur propre :

Proposition 2.2 ([Gu04]) La famille $\partial \Omega_{p, u}$ vérifie

$$
\lim _{u \rightarrow 0} \mu_{p, 1}\left(\partial \Omega_{p, u}\right)=0
$$


et il existe des constantes $c(n)>0$ et $v(n)>0$ telle que

$$
\mu_{p, 2}\left(\partial \Omega_{p, u}\right) \geq c \text { et } \mu_{q, 1}\left(\partial \Omega_{p, u}\right) \geq c
$$

pour tout $u$ et tout $q \neq p$, et

$$
\operatorname{Vol}\left(\partial \Omega_{p, u}\right)<v
$$

pour tout $u$.

On aura besoin en outre de certaines propriétés de symétrie de $\partial \Omega_{p, u}$. Par construction, le domaine $\Omega_{p, u}$ est invariant sous les actions de $\mathrm{SO}_{p+1}(\mathbb{R})$ sur les $(p+1)$ premières coordonnées de $\mathbb{R}^{n+1}$ et de $\mathrm{SO}_{n-p}(\mathbb{R})$ sur les $(n-p)$ dernières coordonnées. On sait alors que si une valeur propre du laplacien est petite par rapport au diamètre maximal des orbites, les formes propres correspondantes sont invariantes (voir [Ja04], théorème 1.10 et lemme 4.1). Notons $\omega_{p, u}$ la forme propre de $\partial \Omega_{p, u}$ associée à la valeur propre $\mu_{1, p}\left(\partial \Omega_{p, u}\right)$. Si $u$ est suffisamment petit, la forme $\omega_{p, u}$ est donc invariante par $\mathrm{SO}_{p+1}(\mathbb{R})$ et $\mathrm{SO}_{n-p}(\mathbb{R})$. En particulier, sa restriction à la sphère $\partial \Omega_{p, u} \cap \mathbb{R}^{p+1} \times\{0\}$ est nécessairement, à un facteur scalaire non nul près, la forme volume canonique. Si on note $\Upsilon$ l'isométrie de $\mathbb{R}^{n+1}$ définie par $\Upsilon\left(x_{1}, \ldots, x_{n+1}\right)=\left(x_{1}, \ldots, x_{p},-x_{p+1},-x_{p+2}, x_{p+3}, \ldots, x_{n+1}\right)$, on en déduit le

Fait 2.3 La sphère $\partial \Omega_{p, u}$ est invariante par $\Upsilon$ et $\Upsilon^{*} \omega_{p, u}=-\omega_{p, u}$.

Cette propriété de la forme $\omega_{p, u}$ sera un élément clef de la construction de valeurs propres doubles.

\section{Création d'une valeur propre double}

On va maintenant utiliser les outils présentés dans la section précédente pour construire une valeur propre double.

On se donne une variété riemannienne $(M, g)$ de dimension $n \geq 3$ quelconque et un intervalle ouvert $I$ ne rencontrant pas le spectre du laplacien agissant sur les $p$-formes coexactes de $(M, g)$, et on va créer une valeur propre double dans cet intervalle. En appliquant des homothéties aux sphères $\partial \Omega_{p, u}$ de la proposition 2.2, on obtient sur la sphère $S^{n}$ deux métriques $g_{1}$ et $g_{2}$, ayant une valeur propres $\lambda_{i}, i=1,2$ dans l'intervalle $I$ de forme propre $\omega_{i}$, toutes les autres valeurs propres étant plus grandes que $I$. En attachant les sphères $\left(S^{n}, g_{1}\right)$ et $\left(S^{n}, g_{2}\right)$ à $(M, g)$ par des anses fines de rayon $\varepsilon$, on obtient la variété de la figure 1. 


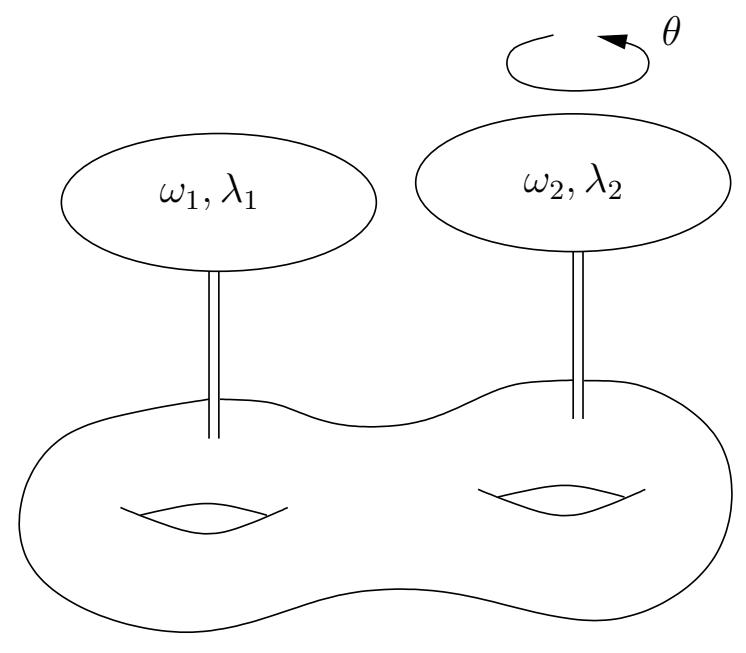

FIG. $1-$

On prend soin de choisir comme point d'attache des anses sur les sphères un point fixe de l'isométrie $\Upsilon$, le recollement entre l'anse et la sphère étant alors déterminé à une isométrie de $S^{n-1}$ près. On fixe cette isométrie arbitrairement sur la $1^{\text {re }}$ sphère, et on se laisse la liberté de faire varier cette isométrie dans un sous-groupe de $\mathrm{SO}(n)$ isomorphe à $S^{1}$ pour la $2^{\mathrm{e}}$ sphère, en notant $\theta$ le paramètre naturel sur le cercle. La métrique ainsi définie sur $M$ est paramétrée par $\lambda_{1}, \lambda_{2}, \varepsilon$ et $\theta$. On peut en outre remarquer qu'on ne modifie pas cette métrique en remplaçant $\theta$ par $\theta+\pi$, du fait de la symétrie de la métrique $g_{2}$ (cf. fait 2.3).

On fixe $\lambda_{1}$ dans l'intervalle $I$ et on choisit une réel $\eta>0$ tel que $\lambda_{1} \pm$ $\eta \in I$. On va créer de la multiplicité en faisant varier $\lambda_{2}$ dans l'intervalle $\left[\lambda_{1}-\eta, \lambda_{1}+\eta\right]$ et $\theta$ dans l'intervalle $[0, \pi]$. On fixe $\varepsilon$ suffisamment petit pour que $M$ n'ait que deux valeurs propres $\mu_{1}$ et $\mu_{2}$ dans l'intervalle $I$, proches de $\lambda_{1}$ et $\lambda_{2}$, et que la somme de leurs espaces propres soit proche de l'espace engendré par $\omega_{1}$ et $\omega_{2}$. Quand les valeurs propres $\mu_{1}$ et $\mu_{2}$ sont distinctes, on posera $\mu_{1}<\mu_{2}$ et on notera $\varphi_{1}$ et $\varphi_{2}$ leurs formes propres respectives. On peut en outre choisir $\varepsilon$ de sorte que si $\lambda_{2}=\lambda_{1}+\eta$ (resp. $\lambda_{1}-\eta$ ), $\varphi_{1}$ est proche de $\omega_{1}$ (resp. $\left.\omega_{2}\right)$. Au final, on ne fera varier la métrique que dans le domaine $D$ de dimension 2 représenté sur la figure 2, et c'est dans ce domaine qu'on va trouver une valeur propre double.

Proposition 3.1 Il existe un point de $D$ pour lequel $\mu_{1}=\mu_{2}$.

Démonstration : L'idée est de considérer la famille à un paramètre de 


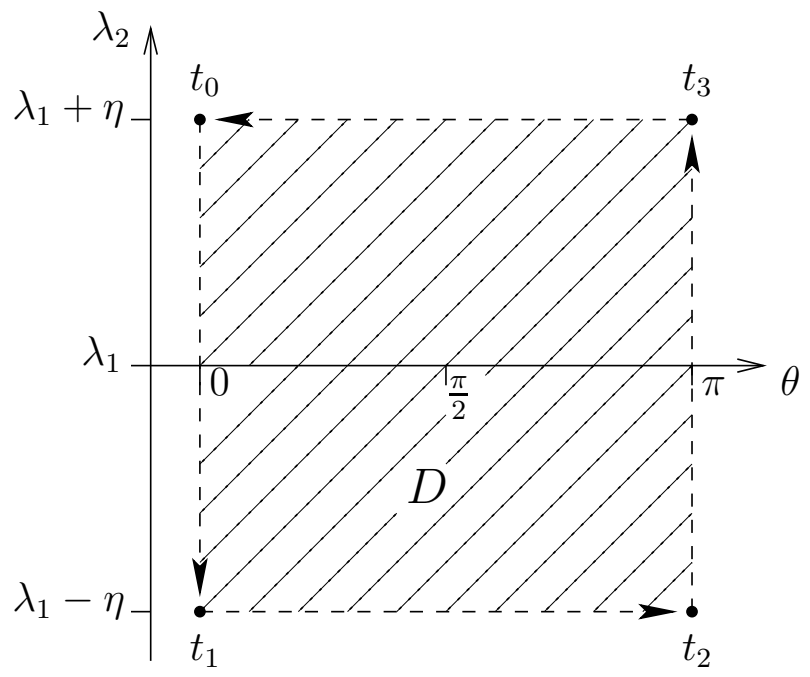

FIG. 2 -

métriques $\left(g_{t}\right)$ obtenue en suivant le bord du domaine $D$ et de suivre l'évolution des $\mu_{i}$ et $\varphi_{i}$. On note $t_{i}$ les valeurs prises par $t$ à chaque coin de $D$ (voir figure 2). Pour toute les métriques paramétrées par $D$, l'espace engendré par les $\varphi_{i}$ est proche de celui engendré par les $\omega_{i}$, il existe donc un isométrie naturelle entre les deux qui permet de les identifier et de représenter les $\varphi_{i}$ et $\omega_{i}$ dans un même plan, comme dans la figure 3

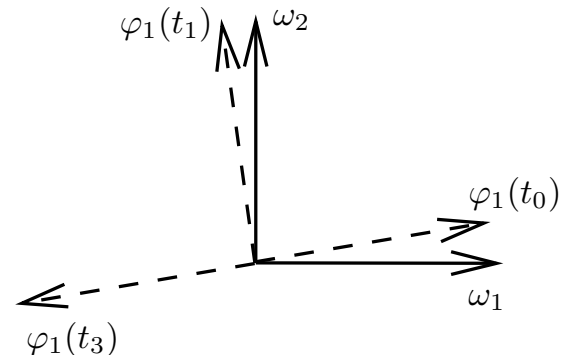

FIG. 3 -

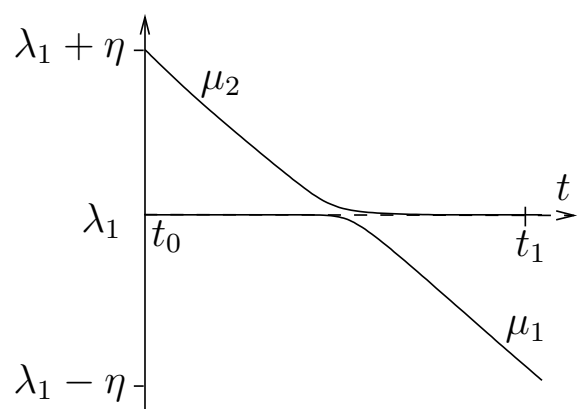

FIG. 4 -

Quand $t=t_{0}$ on a $\lambda_{2}=\lambda_{1}+\eta$, donc $\mu_{1}$ et $\varphi_{1}$ sont proches de $\lambda_{1}$ et $\omega_{1}$ respectivement (voir figures 3 et 4 ).

Quand on passe de $t_{0}$ à $t_{1}$, il est possible - mais improbable - qu'on 
croise un point pour lequel $\mu_{1}=\mu_{2}$. Si c'est le cas, la proposition est démontrée. Sinon, les deux valeurs propres restent distinctes et évoluent comme sur la figure 4: pour $t=t_{1}$, les valeurs propres $\mu_{1}$ et $\mu_{2}$ sont proches de $\lambda_{2}=\lambda_{1}-\eta$ et $\lambda_{1}$ respectivement. La forme $\varphi_{1}$ s'est donc déplacée et est maintenant proche de $\omega_{2}$ comme sur la figure 3 (quitte à changer la convention de signe sur $\left.\omega_{2}\right)$.

On passe ensuite de $t_{1}$ à $t_{2}$, c'est-à-dire qu'on fait tourner la $2^{\mathrm{e}}$ sphère d'un demi-tour autour du point d'attache à l'anse sans modifier $\lambda_{1}$ et $\lambda_{2}$. La forme $\varphi_{1}$ reste donc proche de $\omega_{2}$. Il faut noter que les métriques $g_{t_{1}}$ et $g_{t_{2}}$ sont isométriques, et donc qu'on peut identifier les espaces propres en $t_{1}$ et $t_{2}$, mais que $\omega_{2}$ et $\varphi_{1}$ ont changé de signe par rapport à cette identification (cf. fait 2.3).

On va ensuite de $t_{2}$ à $t_{3}$ en faisant passer $\lambda_{2}$ de $\lambda_{1}-\eta$ à $\lambda_{1}+\eta$. Par rapport au chemin $\left[t_{1}, t_{0}\right]$, la différence est que $\theta$ a varié de $\pi$, c'est-à-dire qu'on passe par des métriques isométriques à celle de $\left[t_{1}, t_{0}\right]$. Les valeurs propres varient donc exactement comme dans la figure 4 en inversant l'axe temporel (en particulier, les valeurs propres $\mu_{1}$ et $\mu_{2}$ restent distinctes). Les espaces propres subissent aussi l'évolution inverse de celle du parcours $t_{0} \rightarrow t_{1}$. Mais $\varphi_{1}$ a changé de signe entre $t_{1}$ et $t_{2}$, en $t_{3}$ c'est donc $-\varphi_{1}$ qui est proche de $\omega_{1}$. Les métriques $g_{t_{0}}$ et $g_{t_{3}}$ sont isométriques, mais la forme $\varphi_{1}$ a changé de signe entre $t_{0}$ et $t_{3}$.

Entre $t_{3}$ et $t_{0}$, la forme $\varphi_{1}$ reste proche de $-\omega_{1}$ puisque $\lambda_{1}$ et $\lambda_{2}$ ne varient pas. Quand la métrique suit le bord de $D$, la forme $\varphi_{1}$ associée à la valeur propre $\mu_{1}$ varie donc continûment jusqu'à prendre finalement, la valeur opposée de sa valeur initiale.

Considérons maintenant le fibré vectoriel trivial au dessus de $D$ de fibre $\mathbb{R}^{2}$, en identifiant la fibre à l'espace engendré par $\omega_{1}$ et $\omega_{2}$, c'est-à-dire au plan représenté par la figure 3. Comme il n'y a pas de multiplicité en restriction à $\partial D$, le sous-fibré en droite, de fibre $\mathbb{R} \varphi_{1}$ est bien défini. On vient de montrer que ce fibré est non orientable.

Supposons maintenant que $\mu_{1} \neq \mu_{2}$ pour toutes les valeurs de $\lambda_{2}$ et $\theta$ dans $D$. Le fibré en droite induit par $\varphi_{1}$ est alors défini sur le domaine $D$ entier. Or, ce domaine est contractile, donc ce fibré est trivial. En particulier, sa restriction à $\partial D$ ne peut pas être non orientable. On en déduit par l'absurde qu'il existe un point à l'intérieur de $D$ pour lequel $\mu_{1}=\mu_{2}$. 


\section{Stabilité de la multiplicité}

Commençons par rappeler la définition des l'hypothèses de transversalité donnée par Y. Colin de Verdière dans [CdV88] pour les valeurs propres multiples (voir aussi la section 3 de [An90]).

On se donne un intervalle $I$ de $\mathbb{R}$, un famille de métrique $g_{a}$, où le paramètre $a$ prend ses valeurs dans un compact $K$ d'une variété, que l'on supposera difféomorphe à une boule, et on suppose que pour une valeur $a_{0}$ du paramètre, on a une unique valeur propre $\lambda_{0}$ du laplacien dans $I$ pour la métrique $g_{0}$, de multiplicité $n_{0}$, et d'espace propre $E_{0}$. Pour $a$ proche de $a_{0}$, la somme $E_{a}$ des espaces propres de valeurs propres contenu dans $I$ est proche de $E_{0}$, ce qui permet de les identifier par une isométrie naturelle (voir CdV88 ou An90 pour sa définition explicite) et on note $q_{a} \in \mathcal{Q}\left(E_{0}\right)$ la forme quadratique ainsi induite sur $E_{0}$ par le laplacien. On note $\Phi: K \rightarrow \mathcal{Q}\left(E_{0}\right)$ l'application $a \mapsto q_{a}$ ainsi définie.

Définition 4.1 La valeur propre $\lambda_{0}$ vérifie l'hypothèse forte (resp. faible) de transversalité si $\Phi$ est une submersion en $a=a_{0}$ (resp. $\Phi$ est essentielle en $\left.a_{0}\right)$.

Comme dans [An90 on dira dans la suite de ce texte qu'une telle valeur propre est fortement (resp. faiblement) stable. La définition d'une application essentielle en $a_{0}$ utilisée dans CdV88 est que si pour tout $\Psi: K \rightarrow \mathcal{Q}\left(E_{0}\right)$ tel que $\|\Psi-\Phi\|_{\infty}<\varepsilon$ on a $\Phi\left(a_{0}\right) \in \Psi(K)$ (nous dirons ici qu'une telle application est métriquement essentielle). unous utiliserons une notion plus forte, que nous nommerons topologiquement essentielle, qui est que la restriction de $\Phi$ à $\partial K$ n'est pas homotopiquement triviale dans $\mathcal{Q}\left(E_{0}\right) \backslash\left\{\Phi\left(a_{0}\right)\right\}$. On peut vérifier que si $\Phi$ est topologiquement essentielle en $a_{0}$ elle est aussi métriquement essentielle, et que si deux applications sont topologiquement essentielles en $a_{0}$ et $b_{0}$ respectivement, alors leur produit est essentiel en $\left(a_{0}, b_{0}\right)$.

Proposition 4.2 La valeur propre double de la proposition 3.1 est faiblement stable.

Démonstration : Dans la section précédente, Le compact $K$ est le domaine $D$, l'espace $E_{0}$ est l'espace engendré par $\omega_{1}$ et $\omega_{2}$ représenté par la figure 3 , Pour normaliser le problème, on se ramène par homothétie à des formes quadratiques sur $E_{0}$ de trace fixée, c'est-à-dire qu'on pose $\mu_{1}+\mu_{2}=c^{t e}$. Cet espace est de dimension 2, on peut l'assimiler à un plan dont un seul point représente une forme quadratique telle que $\mu_{1}=\mu_{2}$. Le reste du plan peut être paramétré en coordonnées polaire par la différence entre les deux valeurs propres et la direction de la droite propre correspondant par exemple à $\mu_{2}$ 
(attention : en faisant tourner la droite propre d'un demi-tour, on revient à la forme quadratique initiale, c'est-à-dire qu'on a fait une tour complet dans le plan des formes quadratiques). En faisant tourner les droites propres comme l'indique la figure 3. on a en fait montré que $\Phi$ envoie $\partial D$ sur une courbe du plan qui entoure la forme quadratique $q_{0}$ de multiplicité 2 , c'est-à-dire que l'indice de la courbe $\Phi(\partial D)$ par rapport à $q_{0}$ est non nul. L'application $\Phi$ est donc topologiquement essentielle en un point de $D$ d'image $q_{0}$, et la multiplicité est faiblement stable (on retrouve au passage l'existence de la valeur propre double). Dans le cas où la multiplicité apparaît sur l'axe $\theta=0$, on al fait disparaître en changeant l'origine due paramètre $\theta$ ou en perturbant la métrique $g$ sur la variété $M$.

Remarque 4.3. La stabilité forte d'une valeur propre multiple est une notion différentielle, alors que la stabilité faible est seulement topologique. Tous les arguments que nous avons utilisé dans cette section et la précédente sont de nature purement topologique, il ne semble donc pas envisageable de montrer que la stabilité est forte par ces moyens.

Remarque 4.4. La stabilité de la multiplicité donnée par la proposition 3.1 peut s'exprimer d'une autre manière : si on déforme légèrement le domaine $D$ dans l'espace des métriques de sorte que le fibré en droite au dessus du bord $\partial D$ reste non trivial, l'argument topologique utilisé reste valable, il y a donc toujours à l'intérieur du domaine un point donnant une valeur propre double.

Remarque 4.5. Une condition nécessaire pour qu'une multiplicité soit stable est que $\operatorname{dim} K \geq \operatorname{dim} \mathcal{Q}\left(E_{0}\right)$ (ou $\operatorname{dim} \mathcal{Q}\left(E_{0}\right)-1$ si on se restreint à des formes quadratiques de trace fixée). En particulier, la dimension de $K$ doit croître au moins quadratiquement par rapport à la multiplicité. La technique que nous avons utilisée pour construire une valeur propre double ne semble donc pas pouvoir se généraliser à des multiplicités supérieures. En effet, si on attache un plus grand nombre de sphères à la variété, le nombre de paramètres disponibles restera une fonction affine du nombre de sphères, même en déplaçant le point d'attache sur la variété et en utilisant tous les degrés de liberté à la jonction des anses.

On peut déduire des résultats obtenus jusqu'ici l'énoncé suivant, qui sera la base de la démonstration du théorème 1.2:

Lemme 4.6 Soit $M$ une variété de dimension $n \geq 3, p$ un entier tel que $1 \leq p \leq \frac{n-1}{2}$ et $C>\nu>0$ et $V>0$ trois réels. Il existe une métrique $g$ sur $M$ telle que 
- la valeur propre $\mu_{p, 1}(M, g)=\nu=\mu_{p, 2}(M, g)$ est de multiplicité 2 et faiblement stable;

- $\mu_{p, 3}(M, g)>C$, et $\mu_{q, 1}(M, g)>C$ pour $1 \leq q \leq \frac{n-1}{2}$ et $q \neq p$;

$-\operatorname{Vol}(M, g)<V$.

Démonstration : On commence par choisir sur $M$ une métrique $g$ telle que $\operatorname{Vol}(M, g)<V / 10$ et $\mu_{q, 1}(M, g)>2 C$ pour tout $q$. On applique ensuite la construction de la section 3 en considérant l'intervalle $I=] \frac{\nu}{2}, \frac{3 \nu}{2}[$, en attachant des sphères de volume inférieur à $V / 10$ et telle que leurs valeurs propres qui ne sont pas dans $I$ soient plus grandes que $2 C$ (la proposition 2.2 le permet) et en choisissant le rayon $\varepsilon$ des anses de sorte que le volume total soit inférieur à $4 V / 10$. Les propositions 3.1 et 4.2 nous disent qu'il y a alors une valeur propre $\mu_{p, 1}(M, g)$ de multiplicité 2 faiblement stable dans l'intervalle $I$. Une homothétie permet de ramener cette valeur propre à $\mu_{p, 1}(M, g)=\nu$ et les conclusions du lemme sont vérifiées.

Remarque 4.7. Le même énoncé pour une valeur propre simple découle immédiatement de la proposition 2.2, la stabilité étant trivialement vérifiée dans ce cas.

\section{Prescription du spectre}

En s'appuyant sur le lemme 4.6 et en utilisant les techniques habituelles de stabilité spectrale, on peut prescrire le début du spectre avec multiplicité 1 ou 2.

Démonstration du théorème 1.2] : On fixe un réel $\delta>0$ tel que

$$
\delta<\inf _{p<k, \nu_{p, i} \neq \nu_{p, j}}\left\{\frac{\left|\nu_{p, i}-\nu_{p, j}\right|}{2}\right\} \text { et } \delta<\frac{V}{2}
$$

et une constante $C>\sup _{p, i} \nu_{p, i}$. On notera $v$ un paramètre variant dans l'intervalle $[V-\delta, V+\delta]$.

Soit $m$ le nombre total de valeur propre à prescrire, tous degrés confondus mais sans compter la multiplicité. Pour chaque $i \leq m$, on note $\nu$ la valeur propre correspondante, on se donne une sphère munie de la métrique donnée par le lemme 4.6 si la valeur propre est double et la remarque 4.7 si elle est simple, avec un volume $v_{i}$ inférieur à $V / 2 m$ : si on note $E_{i}$ l'espace propre correspondant et $q_{i}$ la forme quadratique sur $E_{i}$ de valeur propre $\nu$, il existe un compact $K_{i}$, une famille de métriques $g_{a_{i}}$ paramétrée par $a_{i} \in K_{i}$, un point $\bar{a}_{i} \in K_{i}$ et une application $\Phi_{i}: K_{i} \rightarrow \mathcal{Q}\left(E_{i}\right)$ tels que $\Phi$ soit essentielle en $\bar{a}_{i}$ avec $\Phi_{i}\left(\bar{a}_{i}\right)=q_{i}, \Phi_{i}\left(a_{i}\right)$ étant la forme quadratique 
induite sur $E_{i}$ par le laplacien pour une métrique $g_{a_{i}}$ sur la sphère. On choisit $K_{i}$ suffisamment petit pour que les valeurs propres de $\Phi_{i}\left(a_{i}\right)$ restent dans l'intervalle $I_{i}=[\nu-\delta, \nu+\delta]$. Par définition de $\delta$ les intervalles $I_{i}$ sont tous disjoints.

On munit la variété $M$ d'une métrique $g$ telle que $\operatorname{Vol}(M, g)=v-\sum_{i=1}^{m} v_{i}$ et $\mu_{p, 1}(M, g)>C$ pour $1 \leq p \leq n / 2$ (c'est possible selon [GP95]) et on attache les $m$ sphères précédemment obtenues, munies d'une métrique $g_{a_{i}}$ avec $a_{i} \in K_{i}$, à $(M, g)$ par des anses de rayon $\varepsilon$. On obtient une variété difféomorphe à $M$, munie d'une métrique qu'on notera $g_{\varepsilon}$ et qui dépend non seulement de $\varepsilon$ mais aussi des $a_{i}$.

La convergence du spectre et des espaces propres donnée par le théorème 2.1 permet de définir ainsi une application

$$
\Phi_{\varepsilon}:\left(\prod_{i=1}^{k} K_{i}\right) \times[V-\delta, V+\delta] \rightarrow\left(\prod_{i=1}^{k} \mathcal{Q}\left(E_{i}\right)\right) \times \mathbb{R},
$$

dont les premières composantes sont données par le spectre de $\left(M, g_{\varepsilon}\right)$ et la dernière étant le volume de $M$, qui converge simplement - et donc uniformément d'après le théorème de Dini — vers l'application $\left(\prod_{i=1}^{k} \Phi_{i}\right) \times I d$ qui est topologiquement, donc métriquement, essentielle en $\left(\bar{a}_{1}, \ldots, \bar{a}_{m}, V\right)$. On en déduit que pour $\varepsilon$ suffisamment petit, le début du spectre et le volume souhaités sont donnés par un élément de l'image de $\Phi_{\varepsilon}$ et que les autres valeurs propres sont plus grandes que $C$.

\section{Grandes multiplicités}

Pour finir, on va démontrer le théorème 1.3 Étant donnés $n \geq 4$ et $1 \leq p<n / 2$, on considère une variété $N_{1}$ de dimension 3 et une variété $N_{2}$ de dimension $n-3$ telle que $b_{p}\left(N_{2}\right)>0$, on pose $M=N_{1} \times N_{2}$ et on fixe un entier $k$ quelconque. Comme la variété $N_{1}$ est de dimension 3, on peut la munir d'une métrique $g_{1}$ telle que la première valeur propre non nulle $\mu_{0,1}\left(N_{1}, g_{1}\right)$ du laplacien agissant sur les fonctions soit de multiplicité $k$ (cf. $\mathrm{CdV86}$ ). Une particularité de la dimension 3 est qu'on peut choisir cette métrique de sorte que $\mu_{1,1}\left(N_{1}, g_{1}\right)>\mu_{0,1}\left(N_{1}, g_{1}\right)$. En effet, les métriques qui interviennent dans la construction de la valeur propre multiple sont obtenues par des déformations conformes à partir d'une famille $\left(g_{a}\right)$ de métriques bien choisies, le volume de $\left(N_{1}, g_{1}\right)$ pouvant être arbitrairement petit (voir la partie 4 de CdV86]). Or, selon Ja06a, si pour toute métrique $g$ sur $N_{1}$ on pose $C(g)=\inf _{\theta \in \Omega^{1}\left(N_{1}\right)} \sup _{\mathrm{d} \zeta=0}\left(\|\mathrm{~d} \theta\|_{\frac{3}{2}}^{2} /\|\theta-\zeta\|_{3}^{2}\right)$ où 
$\|\cdot\|_{p}$ désigne la norme $L^{p}$, on a $\mu_{1,1}\left(N_{1}, \tilde{g}\right) \operatorname{Vol}\left(N_{1}, \tilde{g}\right) \geq C(g)$ pour toute métrique $\tilde{g}$ appartenant à la classe conforme de $g$. On peut en outre choisir la famille $\left(g_{a}\right)$ telle qu'elle soit petite pour la distance de Lipschitz. La constante $C(g)$ sera alors clairement minorée sur cette famille, on a donc une minoration uniforme $\mu_{1,1}\left(N_{1}, \tilde{g}\right) \operatorname{Vol}\left(N_{1}, \tilde{g}\right) \geq C>0$ pour toute métrique $\tilde{g}$ conforme à une métrique $g_{a}$, et en particulier on peut choisir $g_{1}$ telle que $\mu_{1,1}\left(N_{1}, g_{1}\right) \geq C / \operatorname{Vol}\left(N_{1}, g_{1}\right)>\mu_{0,1}\left(N_{1}, g_{1}\right)$.

On munit ensuite $N_{2}$ d'une métrique $g_{2}$ telle que le spectre non nul de $\left(N_{2}, g_{2}\right)$ soit plus grand que $\mu_{0,1}\left(N_{1}, g_{1}\right)$. Si $M$ est muni de la métrique produit $g_{M}=g_{1} \oplus g_{2}$, la formule de Künneth nous dit que $\mu_{p, 1}\left(M, g_{M}\right)=$ $\mu_{0,1}\left(N_{1}, g_{1}\right)$, la multiplicité de $\mu_{p, 1}\left(M, g_{M}\right)$ étant au moins égale à celle de $\mu_{0,1}\left(N_{1}, g_{1}\right)$, les produits des relevées des formes propres de $\mu_{0,1}\left(N_{1}, g_{1}\right)$ avec une $p$-forme harmonique de $\left(N_{2}, g_{2}\right)$ étant des formes propres de $\mu_{p, 1}\left(M, g_{M}\right)$.

Remarque 6.1. La multiplicité $k$ étant stable sur $N_{1}$, on peut la transplanter sur la somme connexe de $M$ avec une autre variété à l'aide du théorème 2.1

\section{Références}

[AC95] C. Anné et B. Colbois - « Spectre du laplacien agissant sur les p-formes différentielles et écrasement d'anses », Math. Ann., 303 (3), p. $545-573,1995$.

[An90] C. AnNÉ - « Fonctions propres sur des variétés avec des anses fines, application à la multiplicité », Commun. Partial Differ. Equations, 15 (11), p. 1617-1630, 1990.

[Ar76] V. ARNOL'D - Les méthodes mathématiques de la mécanique classique, Mir, 1976.

[BCC98] G. Besson, B. Colbois et G. Courtois - « Sur la multiplicité de la première valeur propre de l'opérateur de Schrödinger avec champ magnétique sur la sphère $S^{2} »$, Trans. Amer. Math. Soc., 350 (1), p. 331-345, 1998.

[BW84] M. V. BERRY et M. WILKINSON - « Diabolical points in the spectra of triangles », Proc. Roy. Soc. London Ser. A, 392 (1802), p. 15-43, 1984.

[CdV86] Y. Colin DE Verdière - « Sur la multiplicité de la première valeur propre non nulle du laplacien », Comment. Math. Helv., 61 (2), p. 254$270,1986$.

[CdV87] Y. Colin DE VERDiÈRE - « Construction de laplaciens dont une partie finie du spectre est donnée », Ann. scient. Éc. norm. sup., 20 (4), p. 99$615,1987$. 
[CdV88] Y. Colin DE VERdière - « Sur une hypothèse de transversalité d'Arnol'd », Comment. Math. Helv., 63 (2), p. 184-193, 1988.

[CdV98] Y. Colin de Verdière - Spectres de graphes, volume 4 de Cours spécialisés, SMF, 1998.

[CdVT93] Y. Colin de Verdière et N. Torki - « Opérateur de schrödinger avec champ magnétique », Sémin. Théor. Spectr. Géom., 11, p. 9-18, 1993.

[Da05] M. DAHL - « Prescribing eigenvalues of the Dirac operator », Manuscripta math., 118 (2), p. 191-199, 2005, math.DG/0311172.

[GP95] G. Gentile et V. Pagliara - «Riemannian metrics with large first eigenvalue on forms of degree $p »$, Proc. of Am. Math. Soc., 123 (12), p. $3855-3858,1995$.

[Gu04] P. GuÉRini - «Prescription du spectre du laplacien de Hodgede Rham », Ann. scient. Éc. norm. sup., 37 (2), p. 270-303, 2004.

[Ja04] P. JAmmes - «Petites valeurs propres des fibrés principaux en tores », prépublication, 2004, math.DG/0404536

[Ja06a] P. Jammes - « Minoration conforme du spectre du laplacien de Hodgede Rham », prépublication, 2006, math.DG/0604591.

[Ja06b] P. JAmmeS - « Prescription du spectre du laplacien de Hodge-de Rham dans une classe conforme », prépublication, 2006, math.DG/0601738.

Pierre JAMmeS

Université d'Avignon

laboratoire de mathématiques

33 rue Louis Pasteur

F-84000 Avignon

Pierre. Jammes@univ-avignon.fr 